Association for Information Systems AIS Electronic Library (AISeL)

SAIS 2014 Proceedings

Southern (SAIS)

4-14-2014

\title{
An Affect Primary Framework for Privacy Decision Making
}

Easwar Nyshadham

Nova Southeastern University, easwar@nova.edu

Gerald Van Loon

The City University of New York, gvanloon@citytech.cuny.edu

Follow this and additional works at: http://aisel.aisnet.org/sais2014

\section{Recommended Citation}

Nyshadham, Easwar and Van Loon, Gerald, "An Affect Primary Framework for Privacy Decision Making" (2014). SAIS 2014

Proceedings. 27.

http://aisel.aisnet.org/sais2014/27

This material is brought to you by the Southern (SAIS) at AIS Electronic Library (AISeL). It has been accepted for inclusion in SAIS 2014 Proceedings by an authorized administrator of AIS Electronic Library (AISeL). For more information, please contact elibrary@aisnet.org. 


\title{
An Affect Primary Framework For Privacy Decision MAKING
}

\author{
Easwar A. Nyshadham \\ Nova Southeastern University \\ easwar@nova.edu
}

\author{
Gerald Van Loon \\ The City University of New York \\ gvanloon@ citytech.cuny.edu
}

\begin{abstract}
With the growth of networked computing, the role of information security and information privacy risks have received significant attention from IS researchers. Many models of risk in the literature assume that people carefully evaluate the costs and benefits of engaging in risky activities. Recent research shows that automatic judgments of risks (affect) and momentary feelings play a significant role in risk judgment and behavior. In this paper, we critique the calculus-based models of privacy risk and explain how affect and momentary feelings suggest alternate explanations and predictions. A conceptual framework, incorporating automatic affect, deliberation and momentary feelings is proposed for risky decision making. Literature on IS privacy concerns is used to illustrate the framework.
\end{abstract}

\section{Keywords}

Affect, Affect Heuristic, momentary feelings, risk-as-feelings, privacy concern, automatic evaluations, Dual Process models

\section{INTRODUCTION}

Information and communication technologies (ICT) enable people to transact, share information with one another, enable both work and play and are generally indispensable. The growth of ICT has also brought in its wake a large number of risks related to conduct of transactions and loss of private information. Researchers in IS are naturally interested in understanding how people judge such risks, management of risks (both technical and behavioral) and implications for regulation and policy (e.g., privacy policies).

On the question of how people judge risks and how it affects their behavior, a large literature has developed within the IS field. In the specific context of privacy risks, Li (2011) and Xu, Luo, Carroll, and Rosson (2012, see their Appendix A) review several models of privacy concerns. Much of the IS literature on privacy draws on a notion of "privacy calculus," which suggests that people judge the risks and benefits of engaging in a certain behavior and trade off the risks and benefits in a deliberate process. In operationalizing a notion of calculus, IS researchers draw from models, which assume that people carefully reason about the consequences of their decisions (cognitive-consequentialist models, according to Loewenstein, Weber, Hsee, \& Welch, 2001). Most IS theory/models thus draw from the consequentialist perspective, such as Theory of Reasoned Action (TRA), Theory of Planned Behavior (TPB), Health Belief models, Protection Motivation theory (PMT), Multi Attribute Utility Theory (MAUT) etc. We focus on two significant criticisms of the conceptualization of risk/concern in the IS theories/models in privacy context.

In one stream of work, Loewenstein, Weber, Hsee, and Welch (2001) introduced the Risk-as-Feelings (RAF) hypothesis and John, Acquisiti, \& Loewenstein (2011) apply RAF ideas in privacy context. Among other things, the RAF paper introduced the notion of feelings-based versus cognitive-consequentialist models and distinguished between anticipated and anticipatory emotions, thus clarifying the thinking of risk researchers. The central contribution of this paper is that anticipatory feelings, that is, the momentary feelings experienced at the time of decision overwhelm deliberate judgments. Thus, risk judgments are unlikely to predict behavior, when momentary feelings are involved.

The second stream of work critiques the foundations of privacy-calculus models. Slovic and colleagues (Slovic, 2004, 2010; Slovic, Finucane, Peters, \& MacGregor, 2004, 2007; Slovic \& Peters, 2006) argue that people judge an object (in context) intuitively and automatically. The automatic affective evaluation (henceforth automatic affect) precedes all information processing and thus affects beliefs, intentions, judgments and behaviors. Thus measures of privacy concern using self-report instruments are likely to be measuring affect rather than the weight of a particular scale item. This suggests that privacy scales may not be measuring what they plan to measure and thus have serious construct validity issues. (See recent critique by Ganzach, Ellis, Pazy, and Ricci-Saig (2008), of Sitkin and Weingart's (1995) risk scale for lack of construct validity, based on similar arguments.)

A second aspect of automatic affect-based arguments is even more fundamental and undermines the "calculus" aspect of privacy risk research. In an interesting set of papers (Alhakami \& Slovic, 1994; Finucane, Alhakami, Slovic, \& Johnson, 2000; Slovic \& Peters, 2006) show that inducing higher liking (positive affect) leads people to judge risks as low and benefits as high (and vice-versa). Thus, stated risks and benefits collected in various surveys/experiments in IS literature are not 
independent constructs and may simply be different expressions of an automatic feeling. Thus, the case for the existence of a privacy calculus at individual level is considerably weakened.

The distinction between IS models of deliberation, Slovic's (2004) affect model and Loewenstein et al.'s (2001) RAF are summarized in Fig.1. The terms - stimulus, context, judgment, decision, automatic affect and momentary feelings - are assumed to be clear. Model 1 in the figure is prototypical of the IS models and assumes that people deliberate on the cues from the stimulus and context and form a judgment, which in turn affects behavior. Model 2 in the figure suggests that automatic affect, based on cues from stimulus and context, underlies judgments. Model 3 represents the core argument from RAF model and suggests that momentary feelings arising from stimulus in context moderate (and often overwhelm) the influence of judgments on behavior.

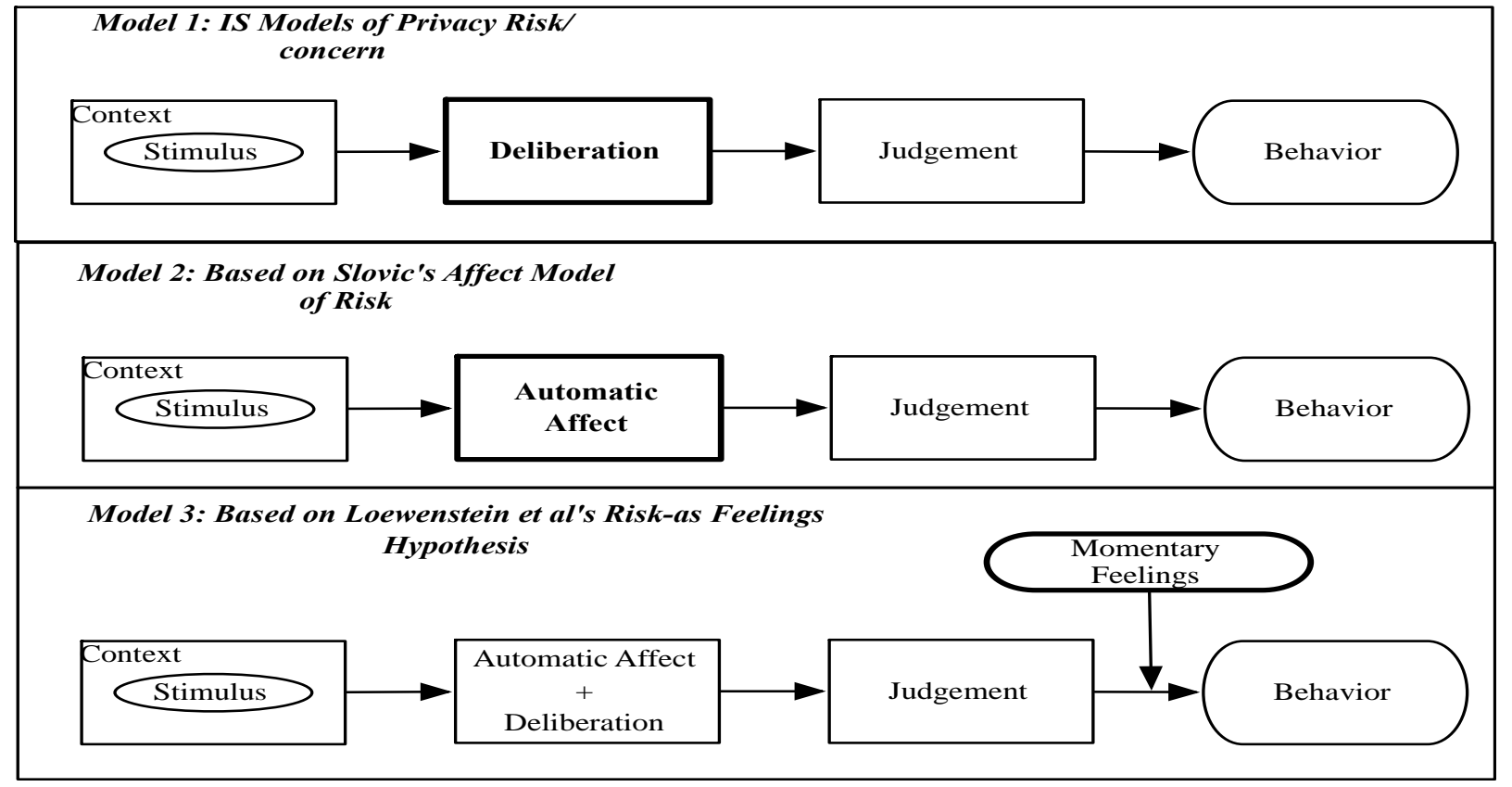

Figure 1. This figure compares model 1, 2, and 3.

The critique of privacy calculus models based on RAF model can be used to explain the privacy paradox, in which privacy attitudes/intentions do not predict privacy behavior. One could fix the current deliberation models in IS by adding, say, a momentary feeling as an additional construct - in the absence of a feeling, deliberate measures may predict behavior whereas in the presence of the feeling, the feeling determines behavior. This may require some redefinitions and re-conceptualizations of existing concepts such as privacy concern to include an anticipatory feeling component. Anderson and Agarwal (2011) in IS and John et al (2011) in a general privacy context are examples of such work.

The critique of privacy calculus models based on the notion of affect, we believe, is much more fundamental. Since automatic affect causes judgments, it raises questions about the appropriate measurement of judgments (and various privacy scales used to measure them) and inferences drawn about attitudes/behavior based on such scales. In cases where automatic affect determines behavior (affect heuristic), deliberation plays no role and even the notion of a calculus comes under question. Thus, the focus of current IS research on identifying different attributes which feed into judgment and estimating model weights (privacy calculus) does not address affect. Under affect arguments, the focus would thus shift to understanding how various cues create an automatic affect, which, in turn affects judgments and behavior.

In summary, the conceptualization of privacy concern in current IS models ignores a) the automatic component of affective evaluation, and b) the dominating role of momentary feelings in explaining privacy behavior. While momentary concerns can be incorporated into deliberation models, it would be much more difficult to incorporate affect into such models without a novel conceptualization of privacy decision making. The purpose of this research is to propose a conceptual framework, which incorporates these considerations. Drawing largely from the work of Kahneman and Frederick (2002, KF henceforth) on decision making in general, this paper suggests an applied framework and several propositions, which can form the basis for theorizing about privacy decision making.

Section 2 discusses several interesting concepts from the KF (2002) framework. In Section 3, a privacy decision making framework based on KF (2002) is proposed and stated as propositions. 


\section{A MODEL OF HEURISTIC JUDGMENT AND SOME CONCEPTS}

KF (2002) propose a model of heuristic judgment for general decision making. The model introduces several concepts such as i) dual process models of reasoning, ii) the idea of natural assessments and affective valence as a natural assessment, iii) attribute substitutability, and iv) cross-dimensional mapping.

A key feature of the model is the idea that two types of cognitive processes operate together in the service of a decision (hence, a dual process model). Of the several dual process models of decision making (See Evans, 2011, for a review), KF (2002) rely on the model proposed by Stanovich and West (2002). Cognitive processes are classified as those which are automatic, rapid and effortless (System 1 processes) and those, which are slow, deliberate and effortful (System 2 processes). System 1 (short for System 1 processes) is involved in constant assessment of internal states and external environment and in producing intuitive judgments, while the occasionally activated System 2 monitors, endorses, corrects and overrides judgments produced by System 1.

KF (2002) suggest that attributes of the stimulus (in context) may be "...routinely evaluated as part of perception and comprehension and therefore always accessible", (p. 271) for judgment. These are called natural assessments, and one such natural assessment is that of judging the affective valence (i.e., degree of liking/disliking for a stimulus in context). The idea of affective valence as a natural assessment in risk context is also fundamental to Slovic's notion of affect, defined as a: "i) specific quality of goodness or badness, ii) experienced as a feeling state (with or without consciousness) and iii) demarcating a positive or negative quality of a stimulus." Affect, per Slovic (2004), is thus an automatic evaluation of a stimulus as good or bad, without deliberation and is similar also to Ferguson and Bargh's (2007) notion of an "object centered context" which is constructed automatically based on cues from stimulus and its context. Thus, we view automatic affect as an evaluation of an object centered context, which is constructed automatically in response to the cues from the stimulus in context.

KF (2002) also discuss a notion of attribute substitution, which suggests that when a person is asked to judge a difficult attribute of a stimulus (e.g., how likely is it that you will be a victim of identity theft?), people use a readily available natural assessment (e.g., how much do I dislike being a victim of ID theft) and substitute it for the target attribute. KF (2002) suggest that people constantly engage in attribute substitution and are often unaware of its effect on their own judgments. The concept of cross-dimensional mapping suggests that when the score of a stimulus on a particular attribute is mapped to another attribute (e.g., people with a dislike for ID theft are asked to judge susceptibility to ID theft), several biases could arise.

The System 1/ System 2 processes in the KF (2002) model are different from popular dual-process models in the IS research such as the Elaboration Likelihood Model (ELM) by Petty, Caccioppo and Schuman (1983) and the Heuristic-Systematic Model (HSM) by Chaiken (1980). The key distinction is that both the heuristic processing mode and the systematic processing mode consume attention and involve cognitive effort - thus, both heuristic and systematic processes are best classified as System 2 processes (see Evans, 2011, p. 268-269 and Bargh, 2002, p. 280-281). System 1/ System2 distinction is based on a default-interventionist view, where System 1 is the automatic, default process guiding reason, while System 2 occasionally intervenes. In contrast, HSM-type models posit two System 2 processes, one involving lower cognitive effort (heuristic) and the other involving higher effort (systematic). The KF (2002) model is thus a default-interventionist view of decision.

\section{A FRAMEWORK OF PRIVACY DECISION MAKING}

Relying on the literature briefly reviewed above, we propose a conceptual framework, which closely follows KF (2002) model of heuristic judgment, as Figure 2 below. In order to communicate the core ideas clearly, we present the framework in a graphical format even though one is dealing with dynamic processes, and suggest specific propositions. Specific research models may test a subset of propositions after suitable contextualization and construct development.

The general argument is summarized as follows. We assume that affect is automatic and a default assessment - thus, affective evaluation of a risky stimulus always takes place, even if a person is unaware of it. When a person is required to judge a difficult target attribute (e.g., how likely is identity theft), that person would often use the readily available affective judgment (e.g., degree of pleasant/unpleasant feeling). People may sometimes experience a meta-cognitive feeling which suggests that something is not quite right, in which case they question their reliance on the automatic assessment and instead engage in effortful deliberation. Once engaged, careful deliberation may endorse, correct or override outputs of automatic affective judgments. Overall, the default risk judgments are based on automatic affect, unless they are endorsed/corrected/overridden by deliberation.

The context of the decision task, defined loosely as everything but the focal hazard, influences risk judgment in two ways. First, the cues from the context magnify or diminish the automatic affect based on a constructive, "object-centered context" view of automatic evaluation (Ferguson \& Bargh, 2007). Thus, marginal changes in context may result in widely different automatic affect being reported. Second, the context contributes to a meta cognitive feeling of fluency/cognitive ease (versus disfluency /cognitive strain). If the decision context creates feelings of fluency/cognitive ease, people rely on automatic affect Proceedings of the Southern Association for Information Systems Conference, Macon, GA, USA March $21^{\text {st }}-22^{\text {nd }}, 2014$ 
- on the other hand, if people experience disfluency / cognitive strain, they would engage in careful deliberation. Thus, deliberation is not a given (unlike current IS models on privacy).

In the interest of space, we present this argument in a graphical form in Figure 2, and state the argument as propositions followed by a brief justification for each proposition.

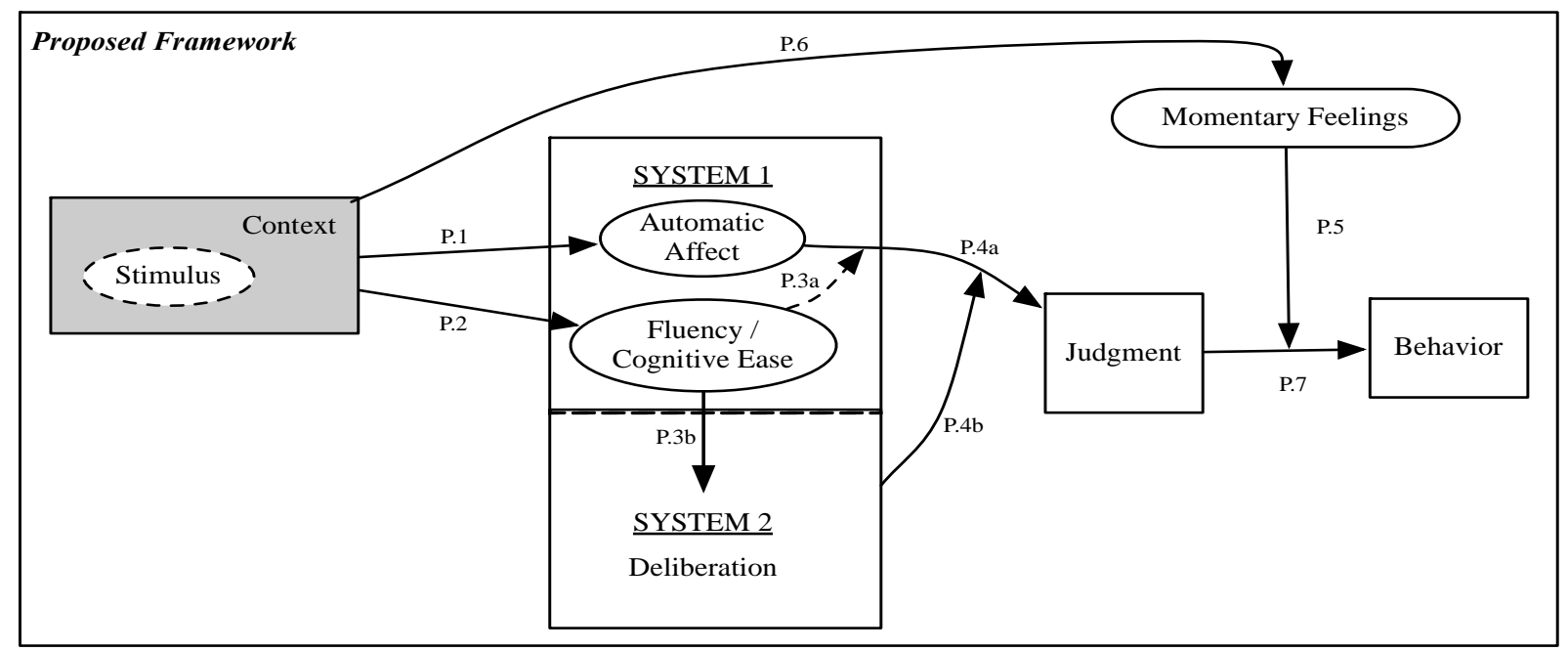

Figure 2. Proposed Framework

Proposition 1: A hazard in context creates an automatic affect in a subject.

Proposition 1 is supported by Slovic and colleagues, and is illustrated and explained in IS context in Nyshadham and Castano (2012) and Nyshadham and Minton (2013). Slovic and colleagues rely on the earlier work of Zajonc (1980) in discussing the idea that affective feelings of good versus bad toward stimuli (natural assessments per KF (2002)) do not need effortful thought - that is, preferences need no inferences. Citing Damasio (1994), they also suggest that images brought to mind are tagged with affect, based on prior experience. The net affect experienced by a person in response to a hazard is thus a combination of tagged affect from component images in the activated network. In the literature on automatic evaluations, Ferguson and Bargh (2007) view automatic evaluations as object-centered contexts, which are constructed spontaneously (as opposed to mere object evaluations, Fazio (2007)). Furthermore, spontaneous evaluation of a stimulus may not require preexisting evaluations (e.g., pre-existing attitudes, prior beliefs, or even tagged imagery) - see Duckworth, Bargh, Garcia, and Chaiken (2002) on the automatic evaluation of novel stimuli. For the purpose of this research, we assume that automatic affect as a primitive evaluation that happens automatically (regardless of the underlying psychological processes) and precedes all information processing. We use the term automatic affect to refer to this primitive evaluation.

As an example, consider a scenario in which a subject is exposed to a nonsense word called Bolobi. Slovic's (2004) argument would suggest that a person would experience a positive/negative feeling on exposure to the nonsense word. If the nonsense word was described with context, say, the Bolobi Virus, people might retrieve imagery corresponding to health issues and may report a different level of automatic affect. Finally, assume that the Bolobi virus is described as a computer virus - then a different set of images may contribute to the experience of automatic affect. In all cases, there is a feeling that is experienced immediately on exposure to the stimulus in context leading to an automatic affect, and will depend on the cues from both the stimulus and the context.

Proposition 1.a: Context can magnify or diminish the experienced affect due to the hazard

One way in which context can influence automatic affect is in an indirect sense. Using the terminology of Pham (2007), one could distinguish between integral affect (feelings attributable to the stimulus/hazard) and incidental affect (in this paper, feelings attributable to the context). When judging risk in context, a person may not be able to distinguish between incidental and integral feelings - and both contribute to automatic affect. Thus, contextual cues are just as important in creating automatic affect as cues from the stimulus.

A second and more interesting explanation uses the notion of affective evaluability of a cue and has been proposed as a mechanism mediating the role of affect in judgments. Bateman, Dent, Peters, Slovic, and Starmer (2007) suggest that the more precise one's affective valuation, the higher the weight it receives in judgment. They explain people's unusual choices in a well-known experiment by Hsee (1996), in which subjects were asked to judge willingness to pay (WTP) for two stimuli (A: a torn dictionary with 20,000 entries and B: a new dictionary with 10,000 entries). In a joint condition in which subjects were asked to provide a WTP for each dictionary, WTP was high for B. In a separate valuation condition, some subjects 
judged WTP for A, while others judged WTP for B and the mean WTP was higher for A. In terms of how good versus bad a cue is, torn versus new cue is easier to evaluate than the relative number of entries; and thus explains the preference for new dictionary in the joint condition. In general, the weight placed on a cue can depend on how affectively evaluable it is.

Proposition 2. Context affects cognitive ease.

Cognitive ease (versus cognitive strain) is defined as a meta cognitive assessment of the current experience of a person (Kahneman, 2011, pp 59-69) and thus is anticipatory. When a person experiences cognitive ease, she would find the situation familiar, feels that it is true, feels good and actions seem effortless. Based on Kahneman (2011, p. 60), if a judgment context involves repeated experience, clear display, primed idea and good mood, then more cognitive ease is experienced. Cognitive ease is similar to fluency (Alter Oppenheimer, Epley, \& Eyre, 2007; Alter \& Oppenheimer, 2009) and for the purpose of this paper, the terms fluency and cognitive ease interchangeably. Within the KF (2002) model, System 1 monitors and assesses cognitive ease on a continuous basis.

In most applied studies of IS risk, context is introduced through the instructions supplied to a subject in a survey or experiment. For example, in a study of judgment of risk due to spyware, Bolobi might be introduced to subjects in the instructions as a surreptitious, drive-by download hazard. In our framework, such a description has two distinct effects. The first effect is the focus of Proposition 1 and suggests that the description creates an affective feeling by bringing to mind a collection of "images" tagged with affect, which would then be combined to create an overall automatic affect for the hazard.

The second effect of context is the focus of proposition 2. This proposition suggests that contextual factors affect cognitive ease (versus cognitive strain). For example, if we had used a difficult sounding word to describe the hazard (Yakuhzda virus instead of Bolobi virus), the difficulty in pronouncing Yakuhzda leads to more cognitive strain (see Song \& Schwarz, 2009). Likewise, if a privacy task was presented using a poorly designed web site, then it is much more likely to lead to cognitive strain (Alter et al., 2007).

Proposition 3: Cognitive ease affects deliberation.

In the framework we propose, a subject always experiences automatic affect rapidly, automatically and probably without awareness. Thus, automatic affect is the default judgment unless the analytical System 2 processes are activated. The two propositions below summarize the main ideas from KF (2002) and Alter et al $(2007,2009)$ suggesting how a meta-cognitive experience such as cognitive ease, might lead to activation of System 2 deliberation processes.

Proposition 3a: If cognitive ease is high, then System 2 processes are not activated.

Proposition 3a suggests that, if cognitive ease is high, then people rely on the default automatic affect and use it as a basis for judgment. Judgments of risk will thus simply reflect automatic affect.

Proposition 3b: If cognitive ease is low, then System 2 processes are activated.

Proposition $3 \mathrm{~b}$ suggests that under low cognitive ease, System 2 processes are activated for checking, endorsing or correcting intuitive judgments. This may lead to automatic affective judgments being modified or even replaced with predictions based on System 2 processes.

Proposition 4: Judgment is based on affect, moderated by deliberation.

The extent to which judgments reflect deliberation depends on whether System 2 processes monitor and endorse/correct intuitive judgments based on affect. Two propositions below state it explicitly.

Proposition 4a: If deliberation is not used, then judgments reflect automatic affect.

Proposition 4a can be explained in detail as follows. If System 2 processes are not activated, then feelings/affect will be used solely in judgment of risk. In this case, if a researcher uses, say, self-report scales (e.g., measures of risk or privacy concern, subjective probabilities or subjective losses) to measure risk judgments, the responses to the items will essentially reflect the automatically generated affective feelings (attribute substitution). The survey questions have the effect of asking a person to report the default automatic affect using the meaning and anchors of a different dimension - this should create some additional biases in measurement as well. KF (2002) call this cross-dimensional mapping and discuss some biases (p. 272). This suggests that, reported risk judgments when System 2 is not activated will be the same as automatic affect, save for biases introduced due to cross-dimensional mapping.

We illustrate the issues using a hypothetical example. Assume that, in the context of privacy, a person has an automatic affective feeling for a stimulus (in context), which has been collected on a simple, semantic differential scale that is anchored on unpleasant feeling (-2) and a pleasant feeling (+2) (using, say, some implicit measurement methods). A researcher might ask the subject to report her judgments on privacy risk/concern using two different measures: a) a global measure of willingness to pay (WTP) to avoid the risk anchored on $\$ 0$ at lower end and open at the higher end, and, b) a probability of 
being a victim of privacy loss, anchored on $0 \%$ to $100 \%$. In the absence of deliberation, it is really the automatic affect that is being reported on the WTP-scale as well as probability-scale - thus, the subject will substitute automatic affect for WTP and probability measures (attribute substitution). The scale anchors for WTP and probability are different - while WTP, expressed as a dollar figure, has no upper bound, the probability scale has natural anchors at $0 \%-100 \%$. Precisely what kinds of biases arise when people map their automatic affect to two semantically related scales (WTP, probability) is beyond the scope of this paper (see KF (2002)). We use this example to illustrate that attribute substitution and cross-dimensional mapping are important considerations in understanding privacy scales - the psychophysics behind cross-dimensional mapping are probably much more nuanced.

Proposition 4b: If deliberation is used, then judgments reflect the outputs of deliberation viz., endorsing, correcting or overriding of automatic affect.

Current IS models of privacy essentially contextualize the deliberation models for privacy decision making (e.g., TRA, TPB, Health Belief models, PMT, MAUT) under the umbrella term of privacy calculus. Li (2011) and Xu et al (2012, see their Appendix A) review several such models - we do not discuss which specific deliberation model applies to privacy decision making under cognitive strain.

Proposition 5: Momentary feelings at the moment of decision can overwhelm deliberate judgments of risk.

A literal interpretation of the path diagram (Loewenstein et al (2001), Fig. 3 on p. 270) in the RAF paper suggests that people i) judge contextual cues and arrive at a subjective evaluation using a deliberate process, as well as, ii) experience momentary feelings in the process, which do not enter subjective evaluations. Under RAF, the momentary feelings overwhelm deliberate evaluations. In John et al. (2011), feelings are operationalized as privacy concern and subjective (objective) hazards are operationalized as product of deliberation. Their work shows that privacy concerns so defined overwhelm deliberation. As we mentioned earlier, incorporating momentary feelings into privacy decision making can explain privacy paradox (for an attempt to explain privacy paradox in terms of the well known intention-behavior discrepancy, see Wells and Valacich (2012)).

The next two propositions do not require much elaboration - proposition 6 is a restatement of the RAF arguments and P7 is well known.

P6. Contextual cues create momentary feelings.

\section{P7. Judgment influences behavior.}

Summary: Risk accompanies most IS decisions - however, most IS research focuses on a narrow conception of risk as deliberation. Two streams of work inform the notion of risk as a feeling. While RAF-based models focus on anticipatory feelings of risk and its ability to overwhelm deliberate judgments of risk, affect-based notions of risk rely on automatic affective evaluations of contextual cues as predecessors of risk judgments. In this paper, we draw upon a dual-system model proposed for general decision making and present a new conceptual framework which incorporates automatic affect, deliberation and anticipatory feelings. In the proposed framework, deliberation and notions of "calculus" play a relatively minor role in explaining privacy decision making. Clarifying and and operationalizing the ideas discussed in the conceptual framework can enhance the understanding of how risk judgments are made by people and how they can be improved.

\section{REFERENCES}

Alhakami, A. S., \& Slovic, P. (1994). A psychological study of the inverse relationship between perceived risk and perceived benefit. Risk Analysis, 14(6), 1085-1096.

Alter, A.L., and Oppenheimer D.M. (2009). Suppressing secrecy through metacognitive ease: Cognitive fluency encourages self-disclosure. Psychological Science, 20(11), 1414-1420.

Alter, A.L., Oppenheimer, D.M, Epley, N, and Eyre, R.N. (2007). Overcoming intuition: Metacognitive difficulty activates analytic reasoning. Journal of Experimental Psychology, 36(4), 569-576.

Anderson C.L., and Agarwal R. (2011). The digitization of healthcare: Boundary risks, emotion, and consumer willingness to disclose personal health information. Information Systems Research, 22(3), 469-490

Bargh, J. A. (2002). Losing consciousness: Automatic influences on consumer judgment, behavior, and motivation. Journal of Consumer Research, 29(2), 280-285.

Bateman, I., Dent, S., Peters, E., Slovic, P., \& Starmer, C. (2007). The affect heuristic and the attractiveness of simple gambles. Journal of Behavioral Decision Making, 20(4), 365-380.

Chaiken, S. (1980). Heuristic versus systematic information processing and the use of source versus message cues in persuasion. Journal of Personality and Social Psychology, 39(5), 752-766.

Damasio, A. R. 1994. Descarte's error: Emotion, reason and the human brain, Avon: New York.

Duckworth K.L, Bargh J.A., Garcia M, and Chaiken S. (2002). The Automatic Evaluation of Novel Stimuli. Psychological Science, 13(6), 513-519

Proceedings of the Southern Association for Information Systems Conference, Macon, GA, USA March $21^{\text {st }}-22^{\text {nd }}, 2014$ 
Evans, J. St. B. T. (2008). Dual-processing accounts of reasoning, judgment and social cognition. Annual Review of Psychology, 59, 255-278

Fazio, R. H. (2007). Attitudes as object-evaluation associations of varying strength. Social Cognition, 25(Special Issue: What is an Attitude?), 603-637.

Ferguson, M. J., \& Bargh, J. A. (2007). Beyond the attitude object: How automatic attitudes spring from object-centeredcontexts. In B. Wittenbrink \& N. Schwarz (Eds.), Implicit measures of attitudes (pp. 216-246). New York: Guilford.

Finucane, M. L., Alhakami, A., Solvic, P., \& Johnson, S. M. (2000). The affect heuristic in judgments of risks and benefits. Journal of Behavioral Decision Making, 13(1), 1-17.

Ganzach, Y., Ellis, S., Pazy, A., \& Ricci-Saig, T. (2008). On the perception and operationalization of risk perception. Judgement and Decision Making, 3, 317-324.

Hsee, C. K. (1996). The evaluability hypothesis: An explanation for preference reversals between joint and separate evaluations of alternatives. Organizational Behavior and Human Performance, 67(3), 247-257.

John, L. K., Acquisiti, A., \& Loewenstein, G. F. (2011). The best of strangers: Context-dependent willingness to divulge personal information. Journal of Consumer Research, 37(5), 858-873.

Kahneman, D., \& Frederick, S. (2002). Representativeness revisited: Attribute substitution in intuitive judgement. In T. Gilovich, D. Griffin, \& D. Kahneman (Eds.), Heuristics \& biases: The psychology of intuitive judgment (pp. 49-81). New York: Cambridge University Press.

Kahneman, D., and Frederick, F. (2005). A Model of Heuristic Judgment. In K. Holyoak \& R.G. Morrison (Eds.), The Cambridge Handbook of Thinking and Reasoning (pp. 267-294). New York: Cambridge University Press.

Li, Y. (2011). Empirical studies on online privacy concerns: literature review and an integrative framework. Communications of the Association for Information Systems, 28, Article 28(1), 453-496.

Loewenstein, G.F., Weber E.U., Hsee, C.K., and Welch, N. 2001. Risk as Feelings. Psychological Bulletin, 127(2), 267-286

Nyshadham, E.A., Castano, D. 2012. Affect and online privacy concerns. Proceedings of the Thirty Third International Conference on Information Systems. Orlando, Florida.

Nyshadham, E., \& Minton, R. (2013). Affect and risk in IS research. Proceedings of the 2013 Southern Association for Information Systems Conference. Paper 29. Macon, Georgia.

Petty, R. E., Cacioppo, J. T., \& Schumann, D. (1983). Central and peripheral routes to advertising effectiveness: The moderating role of involvement. Journal of Consumer Research, 10(2), 135-148.

Pham, M. T. 2007. Emotion and rationality: A critical review of empirical evidence. Review of General Psychology, 11(2), $157-178$.

Song, H., \& Schwarz, N. (2009). If it's difficult to pronounce, it must be risky: Fluency, familiarity, and risk perception. Psychological Science, 20(2), 135-138.

Sitkin, S.B., Weingart, L.R., 1995. Determinants of risky decision-making behavior: a test of the mediating role of risk perceptions and propensity. Academy of Management 38 (6), 1573-1592.

Slovic, P. (2004). What's fear got to do with it? It's affect we need to worry about. Missouri Law Review, 69, 971-990.

Slovic P. (2010). The feeling of risk: New perspectives on risk perception. New York: Earthscan Publishing.

Slovic, P., Finucane, M. L., Peters, E., and MacGregor, D. G. (2004). Risk as analysis and risk as feelings: Some thoughts about affect, reason, risk, and rationality. Risk Analysis, 24(2), 311-322.

Slovic, P., Finucane, M. L., Peters, E., and MacGregor, D. G. 2007. The affect heuristic. European Journal of Operational Research, 177, 1333-1352.

Slovic, P., \& Peters, E. (2006). Risk perception and affect. Current Directions in Psychological Science, 15, 322-325.

Stanovich, K. E., \& West, R. F. (2000). Individual differences in reasoning: Implications for the rationality debate. Behavioral and Brain Sciences, 23, 645-726.

Wilson, D., \& Valacich, J. (2012). Unpacking the privacy paradox: Irrational decision-making with the privacy calculus. Paper presented at the Proceedings of the 33rd International Conference on Information Systems, Orlando.

Xu, H., Luo, X. R., Carroll, J. M., \& Rosson, M. B. (2011). The personalization privacy paradox: An exploratory study of decision making process for location-aware marketing. Decision Support Systems, 51(1), 135-174.

Zajonc, R. B. (1980). Feeling and thinking: Preferences need no inferences. American Psychologist, 35(2), 151-175. 